\title{
The Golden Letter: Innovation Collaboration to Reduce Avoidable Hospital Admissions
}

\author{
Mruga Nanavati, MD, Stephen Saenz, DO, Kiersten Swayne, MD, and \\ Peter Carek, MD, MS
}

Purpose: Emergency Department (ED) overutilization and avoidable hospital admissions have been identified as areas of improvement. Studies have shown that ensuring adequate primary care follow-up after discharge from ED is an area that can be targeted to help decrease hospitalizations.

Methods: Between April 2017 and December 2018, any family medicine patient presented by the ED for admission who was determined to be a candidate for rapid primary care follow-up, was offered a "Golden Letter." This letter guaranteed that the patient would be evaluated within 48 hours of discharge at the patient's primary care clinic. We studied the impact on reducing hospital admissions, 30-day ED revisits, and 30-day hospitalizations. Our data analysis included gender, age, and insurance status.

Results: A total of 191 ED encounters that may have been admitted to the hospital were instead given a golden letter after shared decision making. A total of 104 (54.5\%) completed a follow-up appointment within 48 hours. The 30-day ED revisit rate was 34\% (65 encounters) with $33(31.7 \%)$ of these patients having completed a follow-up visit and $32(36.8 \%)$ did not follow up. There were 35 (18.3\%) hospital admissions within 30 days of the ED visit. Of the patients admitted, 12 (11.5\%) completed a follow-up visit and 23 (26.4\%) did not complete a follow-up visit.

Conclusion: This initiative effectively reduced the number of low-acuity admissions and 30-day hospitalizations. This concept can provide a significant reduction of health care resource utilization and cost by ensuring safe ED discharge and rapid outpatient follow-up for low-acuity patients. ( $\mathrm{J}$ Am Board Fam Med 2020;33:1011-1015.)

Keywords: Aftercare, Family Medicine, Hospital Emergency Service, Hospitalization, Patient Discharge, Quality Improvement, Shared Decision Making

\section{Introduction}

Overall Emergency Department (ED) utilization has increased according to the Center for Disease Control and Prevention with over 145.6 million visits in $2016 .{ }^{1}$ In particular, nonurgent use has increased, with a systemic review showing upwards of $32 \%$ (4.8\% to $90 \%) .{ }^{2,3}$ Although no universal definition is available, nonurgent $\mathrm{ED}$ visits are often defined as those that occur 4 to 72 hours after onset of symptoms or for conditions that can be safely managed on an

This article was externally peer reviewed.

Submitted 19 February 2020; revised 18 May 2020; accepted 19 May 2020.

From the Department of Community Health and Family Medicine, University of Florida, Gainesville, FL (MN, SS, $\mathrm{KS}, \mathrm{PC})$.

Funding: None.

Conflict of interest: None.

Corresponding author: Mruga Nanavati, MD, Department of Community Health and Family Medicine, University of Florida, 1600 SW Archer Road Suite N107, Gainesville, FL 32603 (E-mail: mnanavati@mednet.ucla.edu). outpatient basis. ${ }^{3}$ Nonurgent ED visits can be a source of overcrowding and a cost burden. With nearly $1 / 3$ of the annual health care expenditure in the United States or about $\$ 1$ trillion spent on inpatient hospital care, reducing potentially avoidable or preventable admissions has become an important marker of quality of care provided. ${ }^{4}$ Several studies have identified certain patient characteristics associated with potentially avoidable admissions, such as functional status, access to outpatient care, or time of arrival to the ED. None of these factors significantly affected admission rate. Further study concerning these, and other factors are needed as potentially avoidable admissions account for 1 in 5 admissions. ${ }^{4}$

The disposition of ED patients can be complicated to determine. An important factor in this decisionmaking process is access to prompt outpatient followup with a primary care physician. ${ }^{4,5}$ Patients with reliable outpatient primary care tend to have decreased ED revisits, hospitalizations, and better continuity of 
care. ${ }^{5-7}$ However, very limited data are present on interventions in the ED that use guaranteed primary care follow-up within 24 to 48 hours to successfully prevent an avoidable admission.

In this study, we examined the impact of the "Golden Letter" initiative, an ED-to-primary-care follow-up within 24 to 48 hours, on the disposition and care provided to patients whose acute medical condition did not necessarily require hospitalization. Secondary objectives were the completion rate of follow-up appointments, ED revisits, and subsequent hospitalizations.

\section{Methods}

The Golden Letter initiative was initiated by the Department of Family Medicine through a collaboration with the ED at a large tertiary care, academic institution in the Southeast. The study period was from April 2017 to December 2018. The family medicine department has 6 clinics and sees over 100,000 patient visits annually.

As part of the initiative, if a family medicine patient who presented to the ED possibly needed an admission, the ED provider called the family medicine resident on call. After discussion and shared decision making among the ED physician, family medicine resident, and the patient, those who were determined to benefit from rapid primary care follow-up within 24 to 48 hours instead of hospitalization were given a
"Golden Letter." The Golden Letter guaranteed an appointment at the patient's primary care clinic within 24 to 48 hours (Figure 1). This letter was uploaded to the electronic medical record and given to the patient in the ED with instructions on how to contact the clinic to be seen the next day or within 48 hours if the $\mathrm{ED}$ visit occurred on the weekend. The clinics ensured the patient was seen in the time frame indicated by using open slots, urgent slots, or overbooking if necessary.

Once a Golden Letter was entered into the electronic medical record, the information was captured by the health care coach nurse at family medicine via a report created by Decision Support, a data analysis program. This report was accessed on a secure Web site by a quality analyst who audited the report for specific data points. Data collected from this report included demographic information, follow-up appointment completion, 30-day ED revisits, and 30-day hospitalization. This study was approved by the University of Florida Institutional Review Board.

\section{Statistical Analysis}

Associations between whether a patient attended a follow-up visit after their ED visit and various variables available in the report were assessed using logistic regression. Several patients in the report received multiple Golden Letters for separate encounters in the ED. Logistic regression models were calculated using the generalized estimating equation

Figure 1. Template of a Golden Letter.

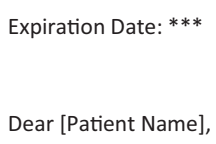


approach to account for correlation between individual patients. All odds ratios were adjusted for the presence of confounders, namely age, gender, and insurance status. Statistical significance was set at $\alpha=0.05 . P$-values were calculated using 2-sided hypothesis tests, using the robust various estimates from the generalized estimating equation model.

\section{Results}

A total of 203 Golden Letters were created and 191 met inclusion criteria (Figure 2). The 12 patients not meeting inclusion criteria were admitted directly from the ED.

Of the subjects, $53(27.7 \%)$ were 65 years of age or older and $115(60.2 \%)$ were female. In terms of insurance status, 22 (11.5\%) had commercial insurance, $13(6.8 \%)$ were insured with Medicare, and 57 (29.8\%) were insured with Medicaid (Table 1). All recipients were established with a primary care physician within our department.

Of the 191 patients that received the Golden Letter and met inclusion criteria, 104 (54.5\%) completed a follow-up appointment with the primary care clinic within 48 hours. The overall 30-day ED revisit rate was $34 \%$ ( 65 encounters). Of note, 33 (31.7\%) of

Figure 2. Total Golden Letters created and outcomes; ED, emergency department.

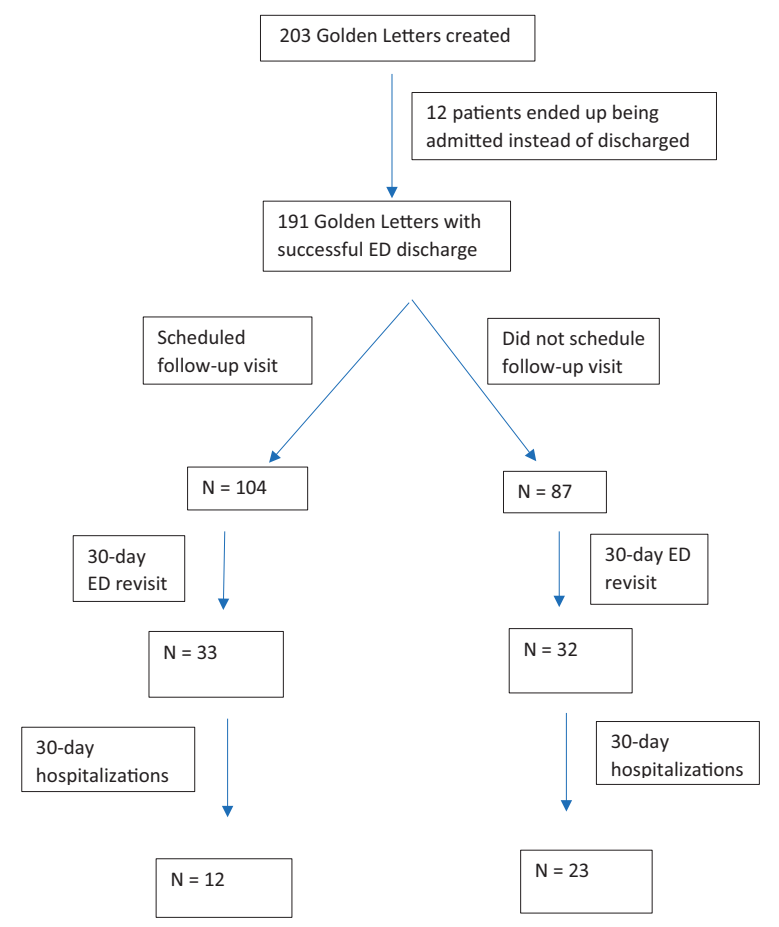

Table 1. Characteristics of Patients Given a Golden Letter in the Emergency Department

\begin{tabular}{lc}
\hline & $\mathrm{N}(\%)$ \\
\hline Total & 191 \\
Demographics & \\
Female & $115(60.2 \%)$ \\
Male & $76(39.8 \%)$ \\
65 years or older & $53(27.7 \%)$ \\
Insurance & \\
Medicaid & $57(29.8 \%)$ \\
Medicare & $13(6.8 \%)$ \\
Commercial & $22(11.5 \%)$ \\
BlueCross & $81(42.4 \%)$ \\
Federal & $2(1.0 \%)$ \\
CMS & $5(2.6 \%)$ \\
Managed care & $11(5.8 \%)$ \\
Follow-up office visit & $104(54.5 \%)$ \\
ED visit within 30 days & $65(34.0 \%)$ \\
Completed follow-up & $33(31.7 \%)$ \\
Did not complete & $32(36.8 \%)$ \\
Inpatient admit within 30 days & $35(18.3 \%)$ \\
Completed follow-up & $12(11.5 \%)$ \\
Did not complete & $23(26.4 \%)$ \\
\hline
\end{tabular}

CMS, centers for medicare \& medicaid; ED, emergency department.

these patients completed a follow-up visit and 32 (36.8\%) did not complete a follow-up visit.

There were 35 (18.3\%) hospital admissions within 30 days of the ED visit. Of the patients admitted, 12 $(11.5 \%)$ completed a follow-up visit and 23 (26.4\%) did not complete a follow-up visit (Figure 2).

Patients' odds of attending a follow-up visit was $78 \%$ (odds ratio $(\mathrm{OR})=0.22 ; 95 \%$ CI, $0.08,0.58$ ) lower among patients who were admitted to inpatient within 30 days of the ED visit compared with those patients who did not complete the recommended follow-up visit. Insurance status (Medicare $[\mathrm{OR}=0.27$; 95\% CI, 0.06, 1.23]; Medicaid OR =0.44; 95\% CI, $0.18,1.04]$, age over 65 years $[\mathrm{OR}=1.97 ; 95 \% \mathrm{CI}$, $0.92,4.25]$ had no impact on odds of presenting for their follow-up appointment (Table 2).

\section{Discussion}

This study examined the ability to have a guaranteed rapid primary care appointment reducing avoidable admissions from ED. As previous studies have shown, no standardized characteristics aid in screening for potentially avoidable admissions. ${ }^{4}$ This initiative relied strictly on clinical judgment 
Table 2. Factors Associated with Attending Follow-up

Odds Ratio (95\% CI)

\begin{tabular}{lc}
\hline Admitted & $022(0.08,0.58)$ \\
ED visit & $1.74(0.78,3.89)$ \\
Over 65 years & $1.97(0.92,4.25)$ \\
Female & $1.04(0.56,1.92)$ \\
Medicare & $0.27(0.06,1.23)$ \\
Medicaid & $0.44(0.18,1.04)$ \\
\hline
\end{tabular}

ED, emergency department; CI, confidence interval.

and shared decision making. This initiative was able to get 191 (94.1\%) patients discharged from the ED instead of likely admission. Twelve (5.9\%) patients were initially deemed appropriate for a Golden Letter, but were later admitted due to worsening clinical status. Despite change in the clinic status of a small number of patients, this initiative provided a significant reduction of health care resource utilization and cost by decreasing hospital admissions for patients who could be safely discharged from the ED. In future studies, it may be beneficial to analyze specific ED diagnoses that are amenable to this model. Current literature does not reveal any specific ED diagnoses that may be studied; however, the Institute of Medicine has a list of "ambulatory care-sensitive conditions" that are often characterized as better suited for outpatient care. ${ }^{8}$ These include chronic obstructive pulmonary disease, congestive heart failure, asthma, hypertension, cellulitis, hypoglycemia, gastroenteritis, dehydration, and dental conditions.

The outpatient follow-up rate was $54.5 \%$. We had several hypotheses as to why it may have been low. For example, the presenting symptom may have resolved or improved within 24 to 48 hours prompting the patient not to follow up or a patient may have gone to an outside health center for care. Several studies have demonstrated $30 \%$ to $50 \%$ follow-up rates in both urban and suburban areas due to various factors such miscommunication or patient perception of urgency of follow-up. ${ }^{9}$ Several ED interventions have been studied to determine their impact on follow-up completion rates, but current literature shows that factors such as older age, evaluation by a consultant in the ED, and establishing a follow-up appointment during the ED encounter are associated with increased follow-up within 7 days. ${ }^{9-13}$ In our model, it is up to the patient to call and schedule the appointment or arrive to clinic in the morning. This added responsibility may be an issue for patients if trans- portation is an issue or other behavioral factors. Our data showed that age greater than 65 years, gender, and insurance status were not associated with higher odds of outpatient follow-up (Table 2). Further study is needed to determine whether other patient characteristic impact follow-up rates.

The overall 30-day ED revisit rate was 34\%, which is greater than the national 30-day ED revisit rate of $19.9 \%$ according to the Health care Cost and Utilization Project. ${ }^{5,14}$ Patients who followed up as recommended during their initial ED visit had a slightly lower revisit rate, but it was not statistically significant. However, primary care follow-up did reduce 30 -day admissions as only $11.5 \%$ of patients who followed up were admitted within 30 days (Table 2). This helps demonstrate the safety of this initiative. However, more can be done to ensure primary care follow-up can to help reduce ED revisits, as the highest revisit rate is within 24 hours after discharge. ${ }^{14}$

\section{Limitations}

Certain limitations are present. The study is a retrospective, nonrandomized trial. While there are acute conditions that disqualify patients for a Golden Letter, there is no standard criteria used in assigning candidacy for a Golden Letter. This lack of standard criteria may potentiate a selection bias and subjectivity. Further, on initial establishment of the Golden Letter initiative, a limitation of 2 letters was allotted per family medicine clinic in a 24-hour period. This restriction may have limited use of additional candidates. In addition, the department was unable to track Golden Letter recipients who may have presented to an ED outside of our institution or any hospitalizations at other institutions. The prevention of admission at our institution does not guarantee that patients were not rendered care at another facility after the letter was given. Lastly, there may be limited generalizability due to this study being conducted at a single institution.

\section{Conclusion}

In conclusion, the Golden Letter initiative was developed to help reduce the number of low-acuity hospital admissions by providing rapid primary care follow-up within 48 hours. This initiative effectively reduced the number of low-acuity admissions and this tool was widely accepted by the hospital and ED providers. As further efforts are made to 
improve this initiative, we hope the current results serve as evidence for other hospitals to establish a similar concept as they address ED admissions and use alternative methods of follow-up that do not include acute hospitalization of the patient.

We would like to acknowledge Kim Lynch for assistance with data report collection and Ben Rooks for assistance with statistical analysis.

To see this article online, please go to: bttp://jabfm.org/content/ 33/6/1011.full.

\section{References}

1. Centers for Disease Control and Prevention. National hospital ambulatory medical care survey: 2016 emergency department summary tables. Published January 19, 2017. Updated 2017. Available from: https://www. cdc.gov/nchs/fastats/emergency-department.htm. Accessed November 4, 2019.

2. Centers for Disease Control and Prevention. National hospital ambulatory medical care survey: 2015 emergency department summary tables. Published 2015. Updated 2016. Available from: https://www.cdc.gov/ nchs/data/nhamcs/web_tables/2015_ed_web_tables.pdf. Accessed November 4, 2019.

3. Durand A, Gentile S, Devictor B, et al. ED patients: how nonurgent are they? Systematic review of the emergency medicine literature. Am J Emerg Med 2011;29:333-45.

4. Daniels LM, Sorita A, Kashiwagi DT, et al. Characterizing potentially preventable admissions: a mixed methods study of rates, associated factors, outcomes, and physician decision-making. J Gen Intern Med 2018;33:737-44.

5. Carmel AS, Steel P, Tanouye R, et al. Rapid primary care follow-up from the ED to reduce avoidable hospital admissions. West J Emerg Med 2017;18:870-7.
6. Sharma G, Fletcher KE, Zhang D, Kuo YF, Freeman JL, Goodwin JS. Continuity of outpatient and inpatient care by primary care physicians for hospitalized older adults. JAMA 2009;301:1671-80.

7. Doran KM, Colucci AC, Hessler RA, et al. An intervention connecting low-acuity emergency department patients with primary care: effect on future primary care linkage. Ann Emerg Med 2013;61:31221.

8. AHRQ Quality Indicators. Guide to prevention quality indicators: hospital admission for ambulatory care sensitive conditions. Rockville, MD: Agency for Healthcare Research and Quality, 2001. AHRQ Pub. No. 02-R0203.

9. Magnusson AR, Hedges J, Vanko M, McCarten K, Moorhead J. Follow-up compliance after emergency department evaluation. Ann Emerg Med 1993;22:560-7.

10. Baren JM, Boudreaux ED, Brenner BE, et al. Randomized controlled trial of emergency department interventions to improve primary care follow-up for patients with acute asthma. Chest 2006;129:257-65.

11. Chan T, Killeen J, Castillo EM, et al. Impact of an internet-based emergency department appointment system to access primary care at safety net community clinics. Ann Emerg Med 2009;54:279_ 84.

12. Kyriacou DN, Handel D, Stein AC, Nelson RR. Brief report: factors affecting outpatient follow-up compliance of emergency department patients. J Gen Intern Med 2005;20:938-42.

13. Katz E, Carrier E, Umscheid C, Pines J. Comparative effectiveness of care coordination interventions in the emergency department: a systematic review. Ann Emerg Med 2012;60:12-23.

14. Duseja R, Bardach NS, Lin GA, et al. Revisit rates and associated costs after an emergency department encounter: a multistate analysis. Ann Intern Med 2015;162:750-6. 